\title{
The Construction of Cognitive Framework of Military English Learning Strategies on the Background of "One Belt, One Road"
}

\author{
He Lixin \\ Amor Technique Institute of PLA \\ Changchun, China \\ Liberty1017@sina.com
}

\author{
Tian Feng \\ Aviation University of Air Force \\ Changchun, China
}

\begin{abstract}
Second Language learning strategy has always been a focused area in English Teaching. By analyzing learning strategies and by looking at the cognitive factors, the paper aims to provide a cognitive framework for military English study. In recent years, the study of military English learning strategy has been the central topic in the field of military English teaching. However, on the background of "One Belt, One Road", the study of military English learning strategy theory need further complemented and developed. This paper researches on military learning strategy from cognitive perspective hoping use the result to effectively promote military English teaching with the improved strategy.
\end{abstract}

Keywords-military English; learning strategy; language acquisition; cognitive linguistics

\section{INTRODUCTION}

The study of learning strategy has been lasting for more than 30 years and has achieved rich research findings. The achievement got by famous professor Wen Qiufang, Wang Lifei and some foreign researchers like Cohen whose findings laid the solid foundation for the further research in this area[1] [8]. Especially the study of learning strategy achieved in recent years aroused more people's attention. On the background of "One Belt, One Road", besides economy, finance and infrastructure, during the development the initiative also should develop the soft contents like language and culture. Therefore, following with the successively learning strategy research findings, based on previous study, this paper studies military English study strategy in military school from cognitive linguistic perspective in such circumstance.

\section{The ReCognition OF CURRENT LEARNING STRATEGY RESEARCH PROBLEM}

Currently, military English study still uses the advantage of second language learning strategy findings. In learning strategies, there are three kinds of classification: two, three and multi-division classification. Here, two-classification learning strategy is direct and indirect strategy or language study and language acquisition strategy. Three-classification is metacognitive strategy and social strategy. And each of them has sub classification. Double dimension classification is management strategy and language learning strategy which exists in in-put, store and output three phases. With regard to English learning strategy, here are some recent research results:

1. Learning strategy has close relationship with success and failure;

2. There are differences in group and individual difference in learning strategy;

3. Learning strategy has effective research method and can be acquired by training;

\section{Learning strategy is helpful in learning language.}

In terms of military English learning, for the learning strategy, there are some big issues need to be recognized, among which includes definition, classification, strategy and other factors, such as strategy research method, and the effectiveness of strategy training.

\section{A. The Recognition of Definition of Learning Strategy}

According to Professor Wang Lifei and Wen Qiufang, the current study mixes the thinking and behavior relationship, that is, the performance and psychology behavior relationship is not so clear[7]. The current definition belongs to both cognitive sphere and motion behavior. The detailed information of clear strategy should be clarified. According to O’Malley \& Channot, learning strategy is also cognitive but it based on other mode types [5]. There are Channot other strategy which is so extensive. The current learning strategy has the problem of size-abstractness dilemma [1]. All these problems increase the difficulty for using the definition. The term of strategy is vague. Oxford, Rubin, Alexander, Graham \& Harris, all of them give the definitions which are different to some extent [4].

\section{B. The Recognition of the Relationship between Learning Strategy and Language Skills}

Whether has learning strategy the positive function for the promotion of language skills? What language strategy can improve the language skills is unknown. That is because the extensive application of learning strategy. According to Qian Yulian, learning strategy does not mean language skills [6]. Language skills is the specific approach which can solve problem during study and can make the study process is more efficient. There is no clear research show there is intimate 
relationship between language strategy and the successful language skill application. According to Rees Miler without clear evidence show there is no obvious direct relationship between learning strategy and language skills. So how is the learning strategy in military language study performed?

\section{The Recognition of the Effectiveness of the Research Method of Learning Strategy}

The current researches cannot truly reflect the reliability of it. The learning strategy methods are most acquired by description, self-thinking approach, which are not so objective. Meanwhile, some strategy evaluation standards are affected by social culture factors. If the strategy carries to other culture environment, the method may not adapt to it, so the result and conclusion is not universal. In addition, the present studies are static research without tracking the following development, which cannot fully present how the strategy affects the practical procedure. And also is the strategy is applied or fully applied in second language study? In some research, it stress on learning strategy training, in fact, learning strategy itself without superior or inferior, there is no united criterion. Learning strategy is cultivated during the acquiring of mother language. To some extent, whether the strategy develops its function, it depends on the learner's attitude and selfconsciousness. Therefore, the latter training of learning strategy modes is not the most important thing.

\section{The Military ENGlish Demands On the BACKGROUnD OF “ONE BELT, ONE ROAD”}

Following with the pushing of the "One Belt, One Road" initiative, we should keep highly sensitiveness of language culture. Especially for military students who are carrying the duty of keeping peace, military practice and other national defense duties. Military English has its own characteristic and style. It featured by unity, simplicity, and non-substitutability in use. In semantics, the characters are semantic uniqueness, accuracy and meaning special. In form, it extensively adopts abbreviation. In term of these, first, military English need multi knowledge structure. Second, military English need flexible training plan. Third, military English need soldiers have the ability to effectively utter their Chinese voice to make others to hear your voice. They are capable of use language to affect and construct people's thought, to build other people's recognition for china and to promote the image of China.

\section{THE CONSTRUCTION OF COGNITIVE FRAMEWORK OF MiLiTARY ENGLISH LEARNING STRATEGIES}

The central focuses of learning strategy are as following: first, does learning strategy work in the brain or out of brain? Second, what does learning strategy consist in its content, knowledge, purpose and performance or all of the three? Third, is the classification acceptable? Fourth, in the circumstance of study and target, does learning strategy own comprehensive character? Fifth, whether is learning strategy always effective and have promoting function? Sixth, is learning strategy a tool in learning or an interior factor? Seventh, all of the term of strategies is vague. Eight, relationship of strategy, skill and procedure is not clear. Ninth, how can strategy promote language learning successively?
Focusing on the above questions, Macaro propose a new theory framework based on information processing and cognitive psychology. It tries to explain the relationship among learning strategy, second language study and the use of second language[3]. This framework consists of three basic features. One is to describe strategy from learning aims, context and psychology behavior. The next is that strategy is a cognitive processing of conscious language material process. Whether strategy is effective that depends on the modes, task and process of strategy. The last is that strategy is different from subconscious activity, language study process, skill plan and learning style.

\section{A. Relationship between Military English learning strategy and subconscious activity}

Based on the above analyzing and theory, the framework distinguishes learning strategy and subconscious activity of brain. Sub consciousness and neurology are mutual action and leads to a chain of variation. But the language learner has no way to control it. All the process may be affected by the consciously applying strategy, but in the long-time memory it is not affected by individual learner. Although it is vague how to process subconscious activity, but working memory, focus of consciousness, attention, controllability and consciousness are mutually complemented, which received universal agreement. Sub consciousness can enter eternal memory through working memory.

\section{B. Relationship between Military English learning strategy and cognitive factors}

If it is admitted working memory and subconscious activity in eternal memory are mutually influenced, the behavior of strategy should have a start point in working memory. Learning strategy happens in working memory, which can be identified in the relationship among strategy and working memory, extensiveness, abstract of strategy and other strategy, strategy and learning aims, strategy and learning tasks.

Where is strategy located? If learning strategy exists in working memory, it is restricted by limited processing resource. But if without learning strategy, working memory cannot perceive, store, process and unlock information. For extensiveness, it is better to define strategy from thinking angle. The real function of strategy can be well understood through description of strategy from psychological behavior. In learning process, it is the combination of strategy. Therefore, there is no single strategy can perform a complex learning task. The comprehension of strategies is mutually united, if any of them perform bad, the group strategy cannot perform its function. The adjustment of strategy needs higher metacognition. Therefore, meta-cognition of the group adjusts conscious cognitive activity. So it is reasonable to divide strategy into cognition and meta-cognition. Working memory resource is occupied by language through operation. For strategy and learning aims, all human activities are directed by purpose and intention, which depends on learning purpose. According to Locke, learning aims should accord to three conditions: choice and promise, specific and accurate, strivings. The key feature of strategy is having accurate aims. For strategy and learning tasks, the feature is learning setting, 
which is transitivity. Just because the transitivity, the language learners can apply the strategy into second language study, which can strengthen the cognitive relationship between strategy and memory.

\section{Learning Strategy and Military English Learning}

Military English learning process make up by cognitive strategy group and meta-cognitive strategy. They affect mutually. Military English learning process is the same with strategy. But for its quick pace it always be misunderstood by sub-consciousness. But in fact, it is a consciousness activity. The process of language involves in the change from one language state to another or from one phase to another or both. In State-like, the mid-state of language converting can be used by Military English learning. But if completing the whole phrases, all the state-like, and stage-like should be involved. In each state, military language learner can have great controllability. From cognitive framework, working memory can continually activate, which can make the change in vocabulary and syntactic structure. All the changes can promote learning skills development.

\section{Learning strategy and learning motivation}

Learning strategies are mutually affected through motivation and strategic plan. The plan is more extensive than strategy learning aims. The plan is constructed by individual meta-cognitive theory. The combination of meta-cognitive theory and cognitive knowledge and experience makes learner have better explanation and forecast. Having learning aims is a necessary condition for strategy construction. The effective application of strategy can enforce motivation and in verse can guarantee the performance of strategy plan. Of course, selfefficacy is one of the factors that can affect the plan.

\section{E. Learning strategy and military English cognitive learning style}

Learners use the same strategy in different settings, which can be regulated as one definite style. Learning style in learning strategy is a habit in processing information, or can be understood as to choose a group strategy in a definite setting. Learning style is stable because it is an intention and general learning method that is relevant to motivation. The approach is a programmed recognition and meta-cognition strategy group. Therefore, cognitive learning style and motivation affect learning style outside cognition.

\section{F. The Relationship between learning strategy and Military language skills}

Military language skills are one form of second language acquiring process. The skill can be evaluated. The successive use of Military language skills (listening, speaking, reading and writing) can make the self-construction of the strategies which can also promote the development of the skills. The ability can promote the formation of chunks. Without the ability and chunks, working memory should have limitation.

\section{The CONTRIBUtion OF COGNITIVE STRATEgy}

The cognitive strategy construction contributes to English learning as follows:

First, strategy occurs in the working memory in brain and strategy has aims, setting and psychological behavior, which solve the extensiveness and abstract of strategy. Second, military English is also second language study, but it has its own features. In the learning process the strategy group exist the whole process. The special tasks of military English need the variation of the strategies. Third, the successful military English study depends on the adjusting of the strategies not the frequencies. Forth, strategy has the feature of transitivity. Fifth, long-term memory is a strategy in military learning, which can strengthen the use of military English.

\section{CONCLUSION}

The cognitive strategy is the complement and perfect for learning strategy, especially on the background of "One Belt, One Road”, in military school, the effective strategy in military English learning becomes more and more important, which can effectively promote English teaching. In future, the learning strategy should pay great attention to how the performance, learning purpose and settings are realized from conscious cognitive version and how to process military English to effectively use strategy group to activate military English aiming to improve language skills.

\section{REFERENCES}

[1] Channot, A. U. \$El-Dinary, P. B. “Children's Learning Strategies in Language Immersion Classrooms” . Journal, Modern Langugage Journal, pp. 83, 1999

[2] Cohen, A. D. "Style and Strategy-Based Instruction in Second Language learning” vol. Foreign Language Teaching and Research Press, 2006

[3] Macaro, E. Strategies for language Learning and for Language Use. Revising the Theoretical Framework, The Modern Language Journal. 2006(3)

[4] Oxford, R. L. "Language Learning Strategies What Every Teacher Should Know” Journal, Boston: heinle. 1990

[5] O’Malley, J. M. \&Channot, A. U. "Learning Strategies in Sencond Language Acquisition” vol. Cambridge: Cambridge Press. 1990

[6] Qian Yulian, "Learning Strategy Analysis of Second Language Learning” Journal of Nanjing Normal University Sep. 2006(5)

[7] Wang Lifei and Wen Qiufang, "English Learner's Ruels and Characters of Motivation, Thinking, Strategy” Foreign Languge and Their Teaching. Journal, pp. 16-20, 2001(2).

[8] Wang Lifei and Wen Qiufang, "The Contruction and Interpretation of the Cognitive Framework of L2 Learning Strategies” Foreign Languge and Their Teaching. Journal, pp. 16-20, 2009(6). 